\title{
cat.dt: An R package for fast construction of accurate Computerized Adaptive Tests using Decision Trees
}

\author{
by Javier Rodríguez-Cuadrado, Juan C. Laria and David Delgado-Gómez
}

\begin{abstract}
This article introduces the cat.dt package for the creation of Computerized Adaptive Tests (CATs). Unlike existing packages, the cat.dt package represents the CAT in a Decision Tree (DT) structure. This allows building the test before its administration, ensuring that the creation time of the test is independent of the number of participants. Moreover, to accelerate the construction of the tree, the package controls its growth by joining nodes with similar estimations or distributions of the ability level and uses techniques such as message passing and pre-calculations. The constructed tree, as well as the estimation procedure, can be visualized using the graphical tools included in the package. An experiment designed to evaluate its performance shows that the cat.dt package drastically reduces computational time in the creation of CATs without compromising accuracy.
\end{abstract}

\section{Introduction}

Nowadays, there is an increasing interest in the development and application of Computerized Adaptive Tests (CATs). For instance, they are applied in several areas such as psychology (Ma et al., 2017; Mizumoto et al., 2019), education (He and Min, 2017; Wu et al., 2017), or medicine (Michel et al., 2018; Fox et al., 2019). The reason behind their popularity is that CATs can estimate the ability level of a psychological variable of interest in an examinee with greater accuracy than the classical tests by administering a smaller number of items (Weiss, 2004). Besides, the existence of certain mechanisms, such as item exposure control (Georgiadou et al., 2007) limits the leaking of items between participants.

Concisely, CATs are tailored tests. Every item administered to the examinee is chosen from an item bank by employing a selection criterion that considers: i) the answers given by the participant to the items previously administered; ii) the characteristics of such items, and iii) the probabilities provided by a model that relates the responses to each item with its characteristics. The most commonly used criterion is Maximum Fisher Information (MFI) (Zhou and Reckase, 2014; Li et al., 2020), which selects the item that provides the highest information for the current estimate of the ability level. However, this criterion presents several drawbacks. These include item selection bias, large estimation errors at the beginning of the test, high item exposure rates, and content imbalance problems (Ueno, 2013; ZhuoKang and Liu, 2012). Various alternatives have been proposed as attempts for addressing these problems. Among them stand out Minimum Expected Posterior Variance (MEPV) (Van der Linden and Pashley, 2009), Kullback-Leibler Information (Chang and Ying, 1996), and Maximum Likelihood Weighted Information (Veerkamp and Berger, 1997). Although these selection techniques largely solve the aforementioned problems, their high computational cost complicates their practical use.

Decision Trees (DTs) have been proposed to reduce the computational cost in the creation of CATs. Yan et al. (2004) used regression trees to predict the participants' total score. A remarkable feature of this work is the merge of nodes to maintain a sufficient sample size to perform the partitions. Afterward, Ueno and Songmuang (2010) developed an item selection criterion based on mutual information in regression trees. However, unlike CATs based on Item Response Theory (IRT), these works predict the total score of the participant rather than estimate their ability level, which makes it difficult to compare the performance of a participant in two different tests aimed at measuring the same construct. Recently, Delgado-Gómez et al. (2019) mathematically proved the equivalence between IRT-based CATs and DTs when the MEPV item selection criterion is used, proposing the Tree-CAT method, which integrates both methodologies. In their method, each node of the tree contains an item, emerging from it as many branches as the number of the item's possible responses. The examinees progress through the tree according to the responses they provide until reaching the last node, where their final estimate corresponds to the found ability level. The disadvantage of this method is that it requires a high-performance cluster to create the tree. In this regard, the Merged Tree-CAT method (Rodríguez-Cuadrado et al., 2020) extends and improves Tree-CAT, accelerating tree construction by joining nodes with similar estimates or ability level distributions.

Currently, there are several packages oriented to the creation of CATs in R. Among them, we can find catR (Magis et al., 2012; Magis and Barrada, 2017), mirtCAT (Chalmers, 2016), and catIrt (Nydick, 2014). The drawback of these packages is that they create a separate CAT for each examinee, reducing their efficiency. For example, if two individuals provided the same response to the first item, the computations for estimation and selection of the next item would be performed twice, even though the 
result would be the same. Therefore, the shortage of memory in these packages considerably increases computational time, which makes the practical application of CATs difficult, or even impossible, when the item selection criterion is one of the most computationally demanding.

This article describes the cat.dt package in which the Merged Tree-CAT method (RodríguezCuadrado et al., 2020) is implemented. Unlike the existing packages, cat.dt creates the CAT before it is administered to the examinees and stores it in a DT structure. This allows that each time the participant responds to an item, the estimation of their ability level and the selection of the next item to be administered is immediate since it is pre-computed. This differs from the existing packages, in which both calculations are performed during the test administration, making it difficult or even impossible to use computationally expensive item selection criteria such as the MEPV. In this way, the cat.dt package manages to quickly create CATs on a standard personal computer as well as provides accurate estimates of the ability level of each examinee.

The rest of the article is structured as follows. Firstly, it introduces the elements of IRT and CATs used by the Merged Tree-CAT. Next, the functions contained in the package are detailed, and an example of its use is provided. Then, the performance of the cat.dt package is compared to that of the cat $\mathbf{R}$ package. Finally, the article discusses the benefits of the package.

\section{IRT and CATs}

The IRT, on which CATs are based, assumes that a participant's response to an item depends on the ability level of the individual and the characteristics of that item (Richardson, 1936; Lawley, 1943; Tucker, 1946). This relationship is obtained through probabilistic models in which the probability $P_{i k}\left(\theta, \pi_{i}\right)$ that an examinee gives the response $k=1, \ldots, K_{i}$ to an item $i$ depends on their ability level $\theta$ and the parameters of the item $\pi_{i}$.

For polytomous items, the most widespread model for ordered responses is the Graded Response Model (GRM) developed by Samejima (1969):

$$
P_{i k}^{*}\left(\theta, \pi_{i}\right)=\frac{e^{\alpha_{i}\left(\theta-\beta_{i k}\right)}}{1+e^{\alpha_{i}\left(\theta-\beta_{i k}\right)}},
$$

where $\alpha_{i}$ is the discrimination parameter, $\beta_{i k}$ are the difficulty (or location) parameters of each response $k$, and $P_{i k}^{*}\left(\theta, \pi_{i}\right)$ the probability of giving the response $k$ or greater. Therefore $P_{i k}\left(\theta, \pi_{i}\right)=$ $P_{i k}^{*}\left(\theta, \pi_{i}\right)-P_{i k+1}^{*}\left(\theta, \pi_{i}\right)$, being $P_{i 1}^{*}\left(\theta, \pi_{i}\right)=1, \ldots, P_{i K_{i}+1}^{*}\left(\theta, \pi_{i}\right)=0$.

When there is not a particular order in the responses, the most generic model is the Nominal Response Model (NRM), defined by Bock (1972):

$$
P_{i k}\left(\theta, \pi_{i}\right)=\frac{e^{\rho_{i k} \theta+\gamma_{i k}}}{\sum_{r=1}^{K_{i}} e^{\rho_{i r} \theta+\gamma_{i r}}},
$$

being $\rho_{i k}$ and $\gamma_{i k}$ the slope and intercept parameters, respectively, for item $i$ and response $k$.

These probabilistic models are used in the CATs to obtain the estimate of the ability level of the examinee based on their responses. Of all the existing estimation methods, the Expected a Posteriori (EAP) technique is widely used given the simplicity of its calculation and the minimum Mean Squared Error (MSE) of its estimations (Bock and Mislevy, 1982). When the responses $R_{i_{1}}, \ldots, R_{i_{M}}$ of the examinee to the items $i_{i}, \ldots, i_{M}$ are the possible responses $k_{1}, \ldots, k_{M}$ of those items, the estimate $\hat{\theta}$ of the ability level is given by:

$$
\hat{\theta}=\int_{-\infty}^{\infty} \theta f\left(\theta \mid R_{i_{1}}=k_{1}, \ldots, R_{i_{M}}=k_{M}\right) d \theta,
$$

being $f\left(\theta \mid R_{i_{1}}=k_{1}, \ldots, R_{i_{M}}=k_{M}\right)$ the posterior density function given the responses according to Bayes' theorem:

$$
f\left(\theta \mid R_{i_{1}}=k_{1}, \ldots, R_{i_{M}}=k_{M}\right)=\frac{P_{i_{1} k_{1}}\left(\theta, \pi_{i_{1}}\right) \cdots P_{i_{M} k_{M}}\left(\theta, \pi_{i_{M}}\right) f(\theta)}{\int_{-\infty}^{\infty} P_{i_{1} k_{1}}\left(\theta, \pi_{i_{1}}\right) \cdots P_{i_{M} k_{M}}\left(\theta, \pi_{i_{M}}\right) f(\theta) d \theta},
$$

where $f(\theta)$ is the prior density function of the examinee's ability level.

Each time an examinee responds to an item, this ability level estimation is used by the CAT to choose the next item. Among the existing item selection methods, MFI is the most popular. This criterion consists of choosing the item $i^{*}$ that maximizes the Fisher information function $F_{i}(\theta)$ evaluated at the current estimate $\hat{\theta}$ of the ability level. This function is given by (Magis, 2015): 


$$
F_{i}(\theta)=\sum_{k=1}^{K_{i}} \frac{P_{i k}^{\prime}\left(\theta, \pi_{i}\right)^{2}}{P_{i k}\left(\theta, \pi_{i}\right)}-P_{i k}^{\prime \prime}\left(\theta, \pi_{i}\right)
$$

Another criterion to highlight is the MEPV because it has been shown to be equivalent to minimizing the MSE of the estimates of the ability level (Delgado-Gómez et al., 2019). In this case, given the posterior density function $f\left(\theta \mid X_{i_{1}}=k_{1}, \ldots, X_{i_{M}}=k_{M}\right)$ obtained from the responses to the items $i_{1}, \ldots, i_{M}$, the MEPV criterion chooses the item $i^{*}$ that minimizes the function:

$$
E_{i}=\int_{-\infty}^{\infty}\left(\sum_{k=1}^{K_{i}}\left(\theta-\hat{\theta}_{M+1, k}^{i}\right)^{2} P_{i k}\left(\theta, \pi_{i}\right)\right) f\left(\theta \mid R_{i_{1}}=k_{1}, \ldots, R_{i_{M}}=k_{M}\right) d \theta,
$$

being $\hat{\theta}_{M+1, k}^{i}$ the ability level estimation if the examinee's response $R_{M+1}$ is the possible response $k$ of item $i$.

In summary, CATs are constructed as follows. Starting from a prior density $f(\theta)$ and a prior estimate $\theta_{0}$, the first item to be administered to the examinee is selected according to an established criterion. Once a response is given, their ability level is estimated, from which the next item to administer is selected. This process is repeated until a stopping criterion is reached (for example, a predetermined number of items to be administered per participant), being the final estimation of the examinee the one obtained after their last response.

The aforementioned process is structured by the Merged Tree-CAT method in a DT as follows (Rodríguez-Cuadrado et al., 2020). As in the Tree-CAT method, each tree node has an assigned item and an associated estimate based on the responses given to the items assigned to the parent nodes. The novelty of the Merged Tree-CAT method consists of limiting the growth of the tree by joining nodes with similar estimates, accelerating its construction with the least loss of precision in the estimates. Once constructed, the examinee progresses through the tree according to their answers until reaching the last node, where the final estimate of their ability level is found. In addition, the Merged Tree-CAT method incorporates item exposure control by establishing an exposure rate that limits the percentage of participants that are administered with each item, which increases the safety of the test.

The cat.dt package implements the Merged Tree-CAT method. As it will be explained in the following section, each of the items that form the test is chosen using the MFI or MEPV criterion, employing the estimate obtained by the EAP method according to the GRM or NRM model.

\section{The cat.dt package}

This section starts by describing the cat.dt package architecture and its main function CAT_DT. This is followed by a practical example on how to use this package to create CATs structured in DTs, to visualize them, and to obtain estimates of the ability level of participants. Finally, we detail some of the computational features taken into account in the building of the package to increase its efficiency.

The cat.dt package can be installed from CRAN (install . packages ("cat.dt")) or from the development version's GitHub repository https://github.com/jlaria/cat.dt. ${ }^{1}$

\section{cat.dt structure}

The cat.dt package consists of the functions shown in Figure 1, which also displays the dependency relationships between them.

The most relevant functions are the following:

- CAT_DT: Creates the CAT structured in a DT.

- create_level_1: Creates the nodes that conform to the first level of the DT.

- create_levels: Creates the nodes that conform to the levels of the DT (except for the first level).

- join_node: Joins nodes from the same tree level with similar estimations or distributions of the ability level.

- CAT_ability_est : Estimates the ability level of a participant after each response and computes a Bayesian credible interval of the final estimation.

\footnotetext{
${ }^{1}$ This package imports the dependencies Matrix, Rglpk, and ggplot2 for matrix treatment, linear programming,
} and visualization, respectively. 


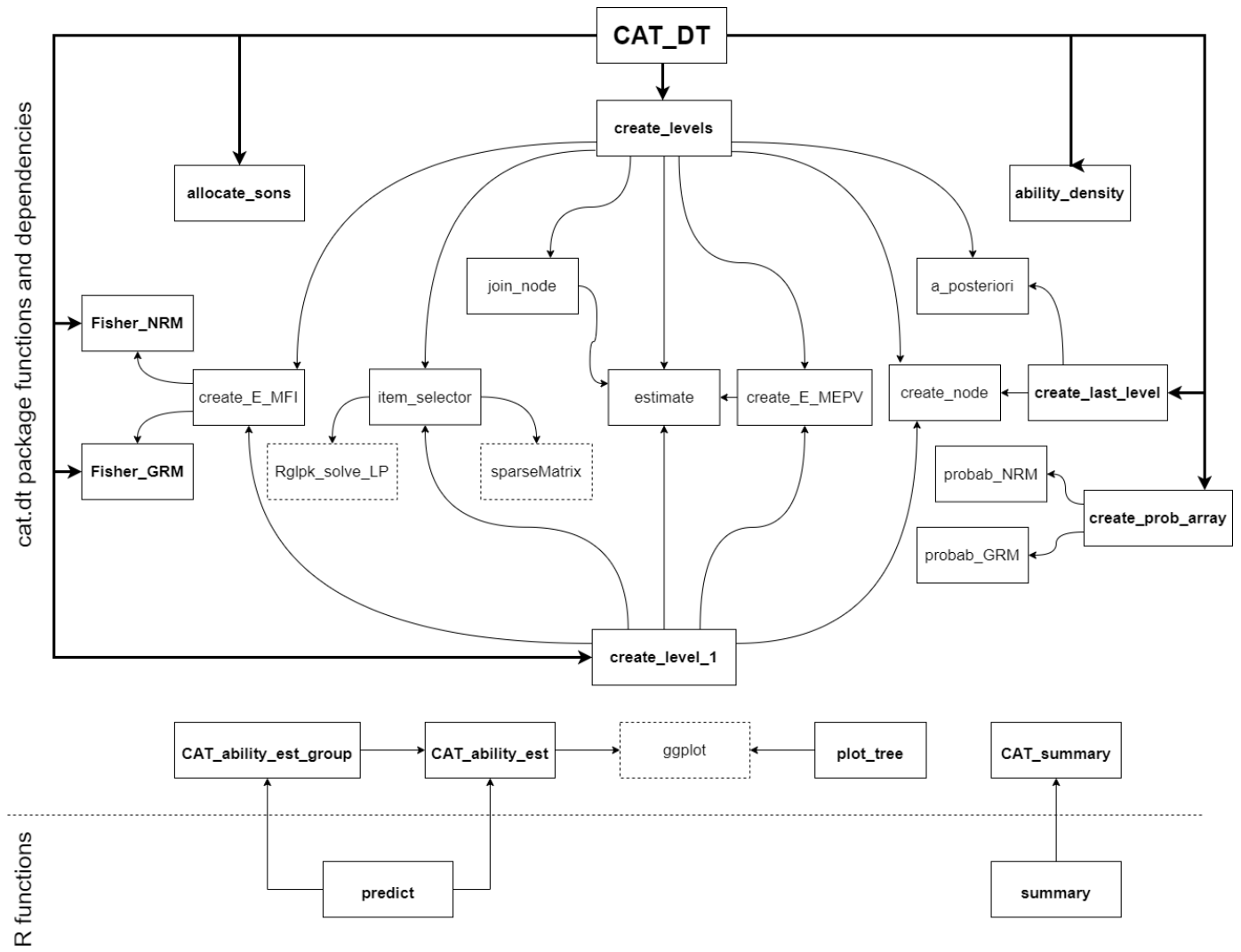

Figure 1: Function dependencies.

\section{The CAT_DT function}

The input parameters of the main function CAT_DT are introduced in Table 1.

The tree growth is controlled by the parameters limit, inters, and $\mathrm{p}$. The limit parameter is the maximum number $N$ of nodes per level. When this number is exceeded in the construction of the tree, those nodes whose estimates of the ability level are at a distance less than a threshold $\left(\lambda_{L}-\lambda_{U}\right) / N$ are joined, being $\lambda_{L}$ and $\lambda_{U}$ the lower and upper bounds of an interval with probability $\mathrm{p}$ according to the prior density function of the ability level. Finally, inters is the minimum value that must exceed the intersection between the density functions of two nodes to join when the maximum number $N$ of nodes per level has not been reached. This intersection is obtained using the methodology defined in Cha (2007).

Finally, the CAT_DT function returns a list with the input parameters introduced by the user and the elements described in Table 2.

\section{cat.dt usage example}

Firstly, it is shown how to build a CAT using the main function CAT_DT. To do this, the item bank from the data frame i temBank included in the package is used. Given the nature of these items, the probabilistic model used is the GRM. Also, the item selection criterion adopted is the MEPV, the exposure rate is set at 0.3 , the length of the test at 10 items, and the prior distribution of the ability level at an $N(0,1)$, leaving the rest of the parameters at their default values. The function call is made as follows:

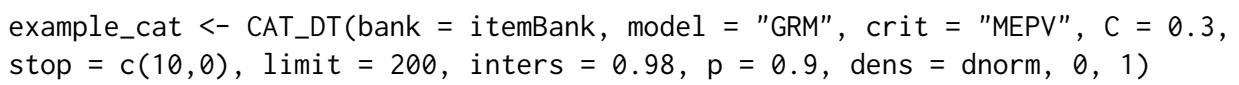

Among the values returned by this function, the list nodes contains all the nodes that conform the DT in which the CAT is structured. These nodes are grouped by levels. As an example, if we access the first node of the third level,

example_cat\$nodes[[3]][[1]]

we obtain 


\begin{tabular}{|c|c|}
\hline bank & $\begin{array}{l}\text { Item bank. It must be a data frame in which each row represents an item and each } \\
\text { column one of its parameters. If the model is GRM, the first column must be the } \\
\text { discrimination parameter and the remaining columns are the difficulty (or location) } \\
\text { parameters (Samejima, 1969). If the model is NRM, the odd columns must be the slope } \\
\text { parameters and the even columns the intercept parameters. (Bock, 1972). }\end{array}$ \\
\hline model & CAT probabilistic model. Options: "GRM" (default) and “NRM". \\
\hline crit & $\begin{array}{l}\text { Item selection criterion. Options: "MEPV" for the Minimum Expected Posterior Variance } \\
\text { (default) or "MFI" for the Maximum Fisher Information. }\end{array}$ \\
\hline C & $\begin{array}{l}\text { Expected fraction } C \text { of participants administered with each item (exposure rate). It can } \\
\text { be a vector with as many elements as items in the bank or a positive number if all the } \\
\text { items have the same rate. Default: } C=0.3 \text {. }\end{array}$ \\
\hline stop & $\begin{array}{l}\text { Vector of two components that represent the CAT stopping criteria. The first component } \\
\text { represents the maximum level } L \text { of the DT and the second represents the threshold for } \\
\text { the Standard Error (SE) of the ability level (Bock and Mislevy, 1982) (if } 0 \text {, this second } \\
\text { criterion is not applied). Default: stop }=c(6,0) \text {. }\end{array}$ \\
\hline limit & $\begin{array}{l}\text { Maximum number } N \text { of nodes per level (max. } N=10000 \text { ). This is the main parameter } \\
\text { that controls the tree growth. It must be a natural number. Default: limit }=200 \text {. }\end{array}$ \\
\hline inters & $\begin{array}{l}\text { Minimum intersection of the density functions of two nodes to be joined. It must be } \\
\text { a number between } 0 \text { and } 1 \text {. If the user wants to avoid using this criteria, inters }=0 \\
\text { should be specified. Default: inters }=0.98 \text {. }\end{array}$ \\
\hline $\mathrm{p}$ & $\begin{array}{l}\text { Prior probability of the interval whose limits determine a threshold for the distance } \\
\text { between estimations of nodes to join. Default: } p=0.9 \text {. }\end{array}$ \\
\hline dens & Prior density function of the ability level. It must be an $\mathrm{R}$ function: dnorm, dunif, etc. \\
\hline$\ldots$ & Parameters to dens. \\
\hline
\end{tabular}

Table 1: Main function parameters.

\$ID

[1] 30001

\$item

[1] 22

\$item_prev

[1] 1811

\$est

[1] -1.10257

\$SE

[1] 0.8071584

\$ID_sons 


\begin{tabular}{|c|c|}
\hline nodes & $\begin{array}{l}\text { List with a maximum of } L+1 \text { elements (levels). Each level contains a list of the nodes } \\
\text { of the corresponding level. Note that the first level will contain more than one root node } \\
\text { if } C<1 \text {. In this case, each examinee would start the test for one of them at random. The } \\
\text { nodes of the additional level } L+1 \text { only include the estimation and distribution of the } \\
\text { ability level given the responses to the items of the final level } L \text {. Note that the nodes } \\
\text { list can have less than } L+1 \text { elements if the SE stopping criterion is satisfied for all the } \\
\text { nodes from a previous level. }\end{array}$ \\
\hline C_left & Residual exposure rate of each item after the CAT construction. \\
\hline predict & $\begin{array}{l}\text { Function that returns the estimated ability level of an examinee after each response and } \\
\text { a Bayesian credible interval of the final estimation given their responses to the items } \\
\text { from the item bank. These responses must be entered by the user as a numeric vector } \\
\text { input. In addition, it returns a vector with the items that have been administered to the } \\
\text { examinee. This is the function CAT_ability_est of the package. }\end{array}$ \\
\hline predict_group & $\begin{array}{l}\text { Function that returns a list whose elements are the returned values of the } \\
\text { function predict for every examinee from the group. This is the function } \\
\text { CAT_ability_est_group of the package. }\end{array}$ \\
\hline
\end{tabular}

Table 2: Main function output.

$\begin{array}{rrrr} & \text { ID_son } & \text { Response } & \text { Probability } \\ 1 & 40001 & 1 & 1 \\ 2 & 40002 & 2 & 1 \\ 3 & 40003 & 3 & 1\end{array}$

\$D

[1] 0.03331851

\$as_val

[1] 0.5396903

This list contains, among others, information about: i) ID, the node identifier; ii) item, the item assigned to the node; iii) i tem_prev, the items previously administered to the examinee that reaches the node; iv) est, the estimation of the ability level after their responses to these items, v) SE, the SE associated to that estimation. Finally, the data frame ID_sons contains the ID of each child node, the response that leads to it, and the probability of, given that response, accessing that child node.

A description of the tree can be obtained using the $\mathrm{R}$ function summary. This function provides: $\mathrm{i}$ ) The number of levels; ii) The number of nodes per level; iii) The probabilistic model used; iv) The item selection criterion used; v) The residual exposure rate of every item and vi) The percentage of items used to build the test. In order to summarize the tree, we enter

summary (example_cat)

to obtain

Number of tree levels: 10

Number of nodes in level $1: 4$

Number of nodes in level 2: 14

Number of nodes in level $3: 39$

Number of nodes in level 4 : 99

Number of nodes in level $5: 101$

Number of nodes in level $6: 124$

Number of nodes in level $7: 141$

Number of nodes in level $8: 158$

Number of nodes in level $9: 165$

Number of nodes in level 10: 177 
Psychometric probabilistic model: GRM

Item selection criterion: MEPV

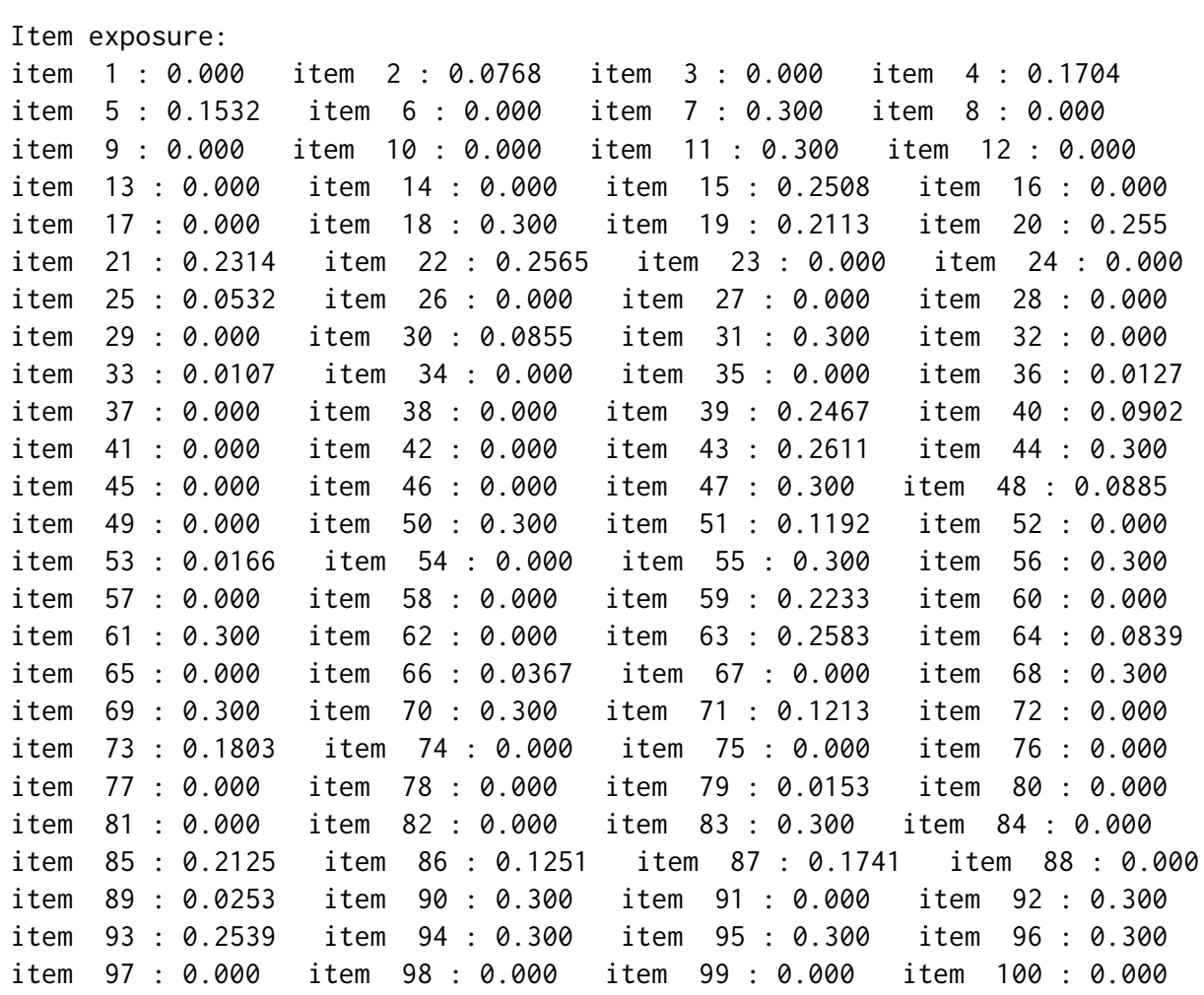

Percentage of items used: $49 \%$

In addition, the tree created can be visualized by means of the function plot_tree. This function takes as input arguments: i) The tree created; ii) The number of levels to plot, iii) The index of the root node to start the test. For example, by introducing

plot_tree (example_cat, levels $=3$, tree $=3$ )

we obtain the following plot

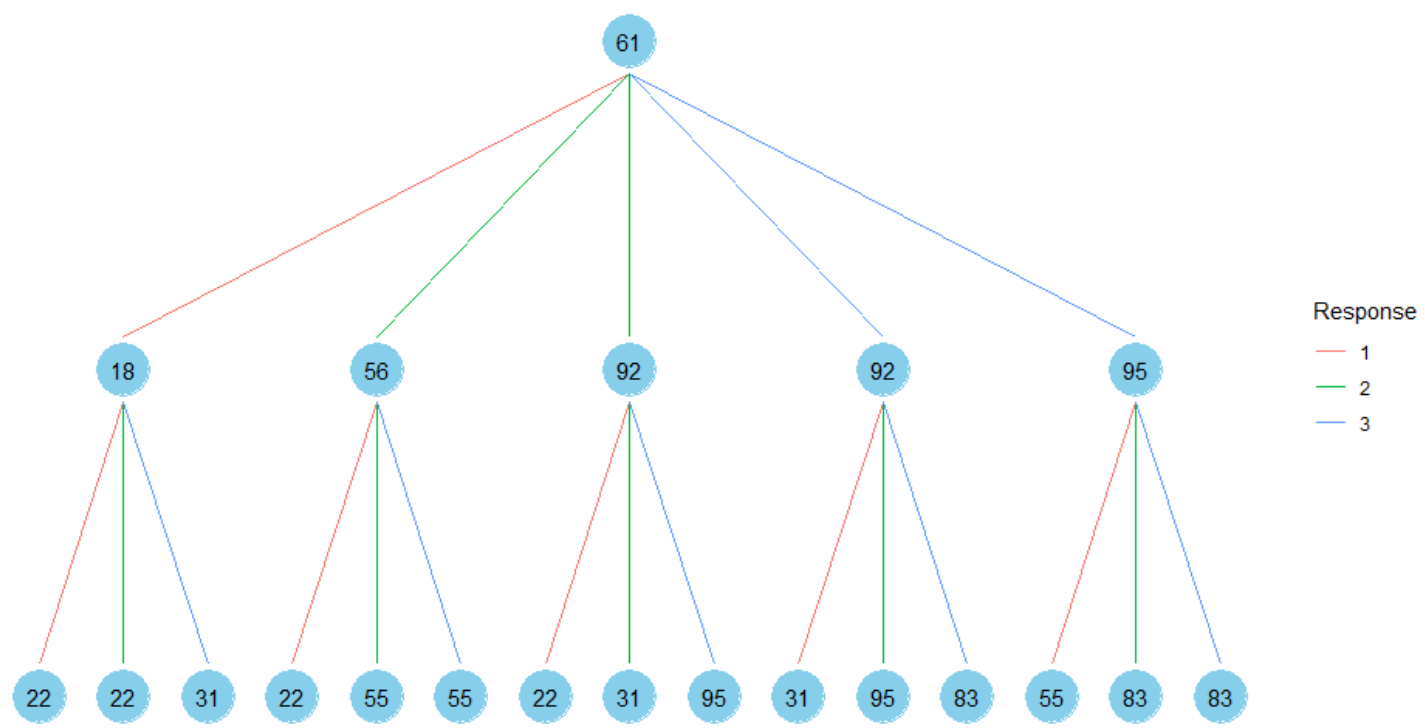

Figure 2: Tree visualization.

Once the CAT has been created, the ability level of an examinee is estimated using the predict 
function. The cat.dt package includes as an example the matrix itemRes, which contains the responses of 30 examinees to the 100 items of the bank itemBank. For instance, to calculate the estimate of the fifth examinee (a seed is needed to obtain the same result due to probabilistic node access) we use

set.seed $(\theta)$

predict (example_cat, itemRes $[5$,$] )$

We obtain

\$estimation-

$\left[\begin{array}{llllllllll}{[1]} & 0.7649071 & 0.3114948 & 0.4320018 & 0.6780359 & 0.4762673 & 0.7993720 & 0.7504451 & 0.5928716\end{array}\right.$

[9] 0.70414790 .6217778

\$1low

[1] -0.325

\$lupp

[1] 1.565

\$items

[1] $\begin{array}{llllllllll}70 & 83 & 95 & 55 & 4 & 39 & 7 & 51 & 96 & 73\end{array}$

\$graphics

This output list contains: i) The vector estimation, which includes the estimated ability level after each response; ii) The lower llow and upper lupp bounds of a Bayesian credible interval at $95 \%$ of the final estimation of the examinee's ability level; iii) The vector i tems, which contains the items that have been administered to the examinee in the CAT, iv) The object graphics, which represents the evolution of the ability level estimation automatically as shown in 3.

\section{Evolution of the ability level estimation}

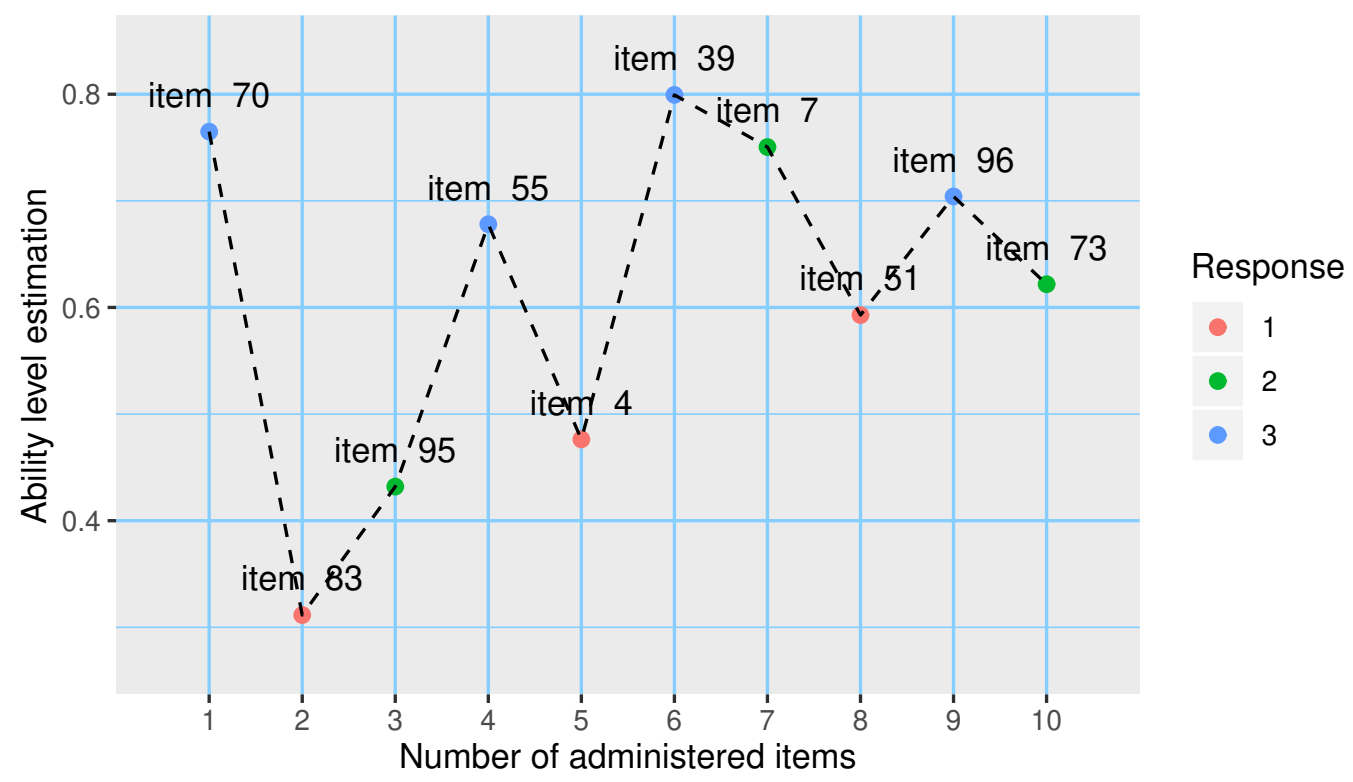

Figure 3: Evolution of the ability level estimation.

This plot represents the estimation of the ability level after responding to each administered item. For example, giving response 3 to item 70 results in an estimate of approximately $\hat{\theta}=0.76$. Then, after giving response 1 to item 83 , the estimate decreases to approximately $\hat{\theta}=0.31$, and so on. Note that the value of the response influences whether the estimate decreases or increases. Alternatively to the predict function, it can be entered CAT_ability_est (example_cat, itemRes $[5$,$] )$ or example_cat\$predict (itemRes $[5$,$] ).$

Finally, these results can be obtained for a whole group of examinees also by calling the function predict. In this case, this function returns a list whose elements are the outputs as if it was called for every examinee. Once it is stored in a variable in the following way,

est_group <- predict(example_cat, itemRes) 
the estimation information is available for every examinee. For instance, to know the items administered to the second examinee, the following must be introduced:

est_group[[2]]\$items

Obtaining:

[1] $\begin{array}{llllllllll}61 & 92 & 95 & 55 & 50 & 59 & 85 & 63 & 19 & 69\end{array}$

Alternatively to the predict function, it can be introduced example_cat\$predict_group(itemRes) or CAT_ability_est_group(example_cat, itemRes).

A similar example can be found in the tutorial vignette included in the package.

\section{Computational features}

Before ending this section, we detail the computational features that accelerate the construction of the tree and reduce memory space.

- Message passing: The calculation of the posterior density function (equation 4 ) is necessary to obtain the estimation of the ability level (equation 3), the selection of the next item according to the MEPV criterion (equation 6), and the Bayesian credible interval of the final estimation. Such calculation would involve the multiplication of $M$ terms for each node of the corresponding level. However, since the multiplication of the first $M-1$ terms is done to obtain the density function of the parent node, this information is stored by the cat.dt package and passed to the child node, in which only the last term is multiplied.

- Joining nodes: the cat.dt package joins those nodes whose estimations and/or posterior density functions meet the similarity criteria determined by the parameters limit, inters, and $p$. These unions control tree growth, which significantly accelerates tree creation and reduces the amount of memory space without losing precision in the estimations.

- Riemann integration and probability pre-calculation: The integrals required in the equations 3,4 , and 6 are approximated numerically by Riemann integration. To do this, a set of ability levels $\left\{\theta_{0}, \ldots, \theta_{4000}\right\}$ is considered, where $\theta_{j}=-10+j / 200$, covering the interval $(-10,10)$. The Riemann integration in equation 6 requires the previous calculation of $P_{i k}\left(\theta_{j}, \pi_{i}\right)$ for each item $i$, possible answer $k$, and ability level $\theta_{j}$. Because of this, these probabilities are calculated and stored before the creation of the tree and then used in equations 4,5 , and 6 . This avoids repeating unnecessary calculations and accelerates the creation of the CAT.

\section{Performance assessment}

In this section, the performance of the cat.dt package is compared to that of the catR package. Ability level estimates and computational times of both packages have been studied for nine different simulation scenarios. In each scenario, a CAT is constructed from an item bank (composed of 100, 200, or 500 items), which is administered to a group of examinees (1000, 2000, or 5000 examinees). The database for each scenario can be found online ${ }^{2}$ in the format [number of items]_items_[number of examinees]_examinees.RData.

Similar to the example of the previous section, the probabilistic model used is GRM, the criteria for selecting items is MEPV, the length of the test is ten items per participant (the SE threshold is set at 0 ), and the prior distribution of the ability level is $N(0,1)$. However, unlike the aforementioned example, there is no item exposure control $(C=1)$ since the implementation differs in both packages: the cat.dt package builds the CAT before administration and the catR package during administration.

All the simulations for both packages were run in an HP Z230 Tower Workstation with an Intel(R) Core(TM) i7-4770 CPU @ 3.40GHz, with 32 GB of RAM, running Debian GNU/Linux 10, R 3.5.2.

Figure 4 illustrates the MSE of the ability level estimates after each response of the examinees, obtained by both packages in each scenario. It is observed that the estimates after each response are equally accurate, indicating that the performance of both packages is very similar.

On the other hand, Table 3 displays the time employed by each package in the computations for the creation of the CAT and the evaluation of those examined in each scenario. It can be seen that the cat.dt package is barely affected by the number of examinees since the CAT is created before it is administered to the participants. However, the catR package creates a CAT for each examinee, so the computational time is proportional to the number of participants. This causes cat $\mathbf{R}$ package to take several days in total for the creation and administration of the CAT, whereas package cat.dt takes a few minutes, being this difference larger the higher the number of examinees.

2https://github.com/jlaria/cat.dt-performance-assessment 


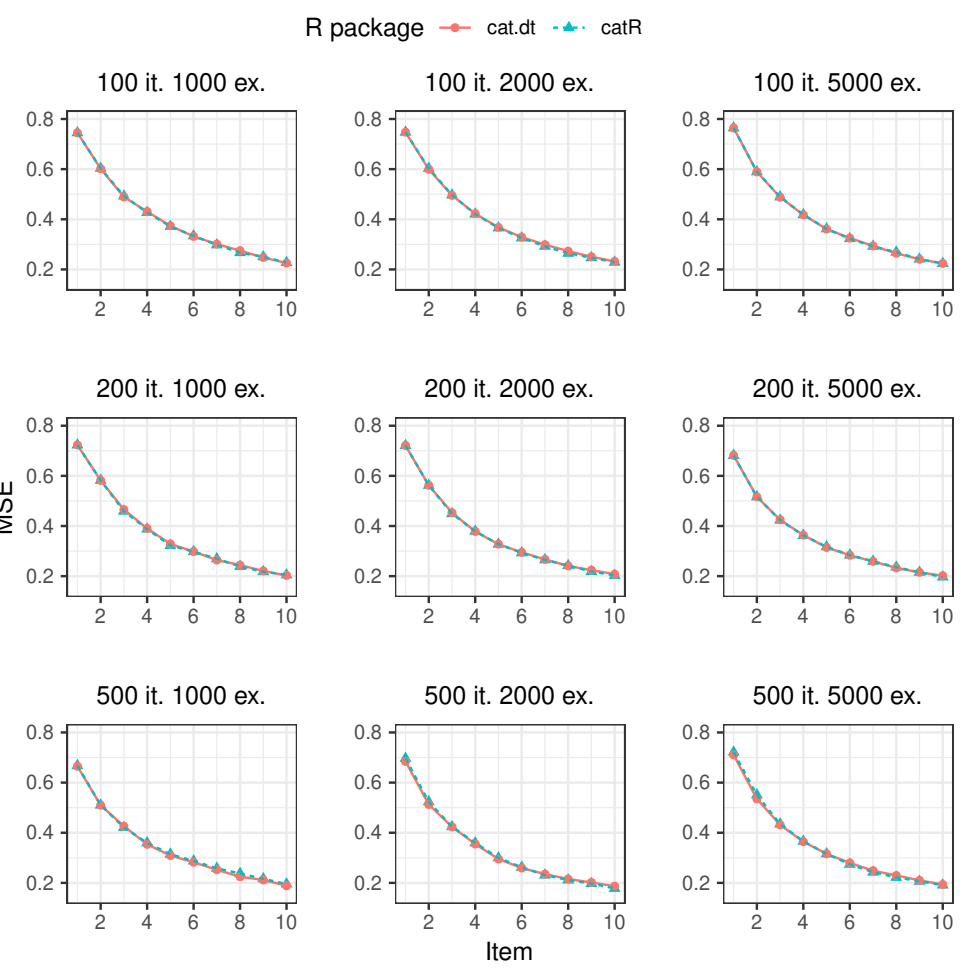

Figure 4: MSE of ability trait estimates.

\begin{tabular}{cccccccc}
\hline & \multicolumn{2}{c}{100 items } & \multicolumn{2}{c}{200 items } & \multicolumn{2}{c}{500 items } \\
\cline { 3 - 8 } & & cat.dt & catR & cat.dt & catR & cat.dt & catR \\
\cline { 3 - 8 } & 1000 & $93.94 \mathrm{~s}$ & $40.23 \mathrm{~h}$ & $263.04 \mathrm{~s}$ & $81.04 \mathrm{~h}$ & $608.71 \mathrm{~s}$ & $206.61 \mathrm{~h}$ \\
$\dot{\Xi}$ & 2000 & $160.67 \mathrm{~s}$ & $78.55 \mathrm{~h}$ & $314.88 \mathrm{~s}$ & $162.22 \mathrm{~h}$ & $635.54 \mathrm{~s}$ & $413.82 \mathrm{~h}$ \\
๘ & 5000 & $160.14 \mathrm{~s}$ & $196.98 \mathrm{~h}$ & $264.50 \mathrm{~s}$ & $406.79 \mathrm{~h}$ & $675.42 \mathrm{~s}$ & $1033.65 \mathrm{~h}$ \\
\hline
\end{tabular}

Table 3: CAT creation and evaluation times.

\section{Summary}

This article has introduced the cat.dt package oriented to the creation of CATs structured in DTs, their visualization, and the estimation of the ability levels of the examinees. Unlike the existing packages, the cat.dt package creates the test before being administered to the examinees, so its performance is independent of the number of participants. For this reason, it is ideal for application to large groups, taking a few minutes to create and administer the test. Besides, it has been shown that the cat.dt package obtains ability level estimates as accurate as those obtained by the catR package, which is widely used in the field of CATs.

\section{Acknowledgments}

This article has been funded by the Spanish National Project No. RTI2018-101857-B-I00.

\section{Bibliography}

R. D. Bock. Estimating item parameters and latent ability when responses are scored in two or more nominal categories. Psychometrika,37(1):29-51, 1972. URL https://doi . org/10.1007/BF02291411. [p124, 127]

R. D. Bock and R. J. Mislevy. Adaptive eap estimation of ability in a microcomputer environ- 
ment. Applied psychological measurement, 6(4):431-444, 1982. URL https://doi.org/10.1177/ 014662168200600405. [p124, 127]

S.-H. Cha. Comprehensive survey on distance/similarity measures between probability density functions. International Journal of Mathematical models and Methods in Applied Sciences, 1(4):300-307, 2007. [p126]

R. P. Chalmers. Generating adaptive and non-adaptive test interfaces for multidimensional item response theory applications. Journal of Statistical Software, 71(5):1-39, 2016. URL https://doi .org/ 10.18637/jss.v071.i05. [p123]

H.-H. Chang and Z. Ying. A global information approach to computerized adaptive testing. Applied Psychological Measurement, 20(3):213-229, 1996. URL https://doi .org/10.1177/014662169602000303. [p123]

D. Delgado-Gómez, J. C. Laria, and D. Ruiz-Hernández. Computerized adaptive test and decision trees: A unifying approach. Expert Systems with Applications, 117:358-366, 2019. URL https: //doi.org/10.1016/j.eswa.2018.09.052. [p123,125]

R. S. Fox, P. I. Moreno, B. Yanez, R. Estabrook, J. Thomas, L. C. Bouchard, H. L. McGinty, D. C. Mohr, M. J. Begale, S. C. Flury, et al. Integrating promis ${ }^{\circledR}$ computerized adaptive tests into a web-based intervention for prostate cancer. Health Psychology, 38(5):403-409, 2019. URL http: //dx.doi.org/10.1037/hea0000672. [p123]

E. G. Georgiadou, E. Triantafillou, and A. A. Economides. A review of item exposure control strategies for computerized adaptive testing developed from 1983 to 2005. The Journal of Technology, Learning and Assessment, 5(8), 2007. [p123]

L. He and S. Min. Development and validation of a computer adaptive efl test. Language Assessment Quarterly, 14(2):160-176, 2017. URL https://doi .org/10.1080/15434303.2016.1162793. [p123]

D. N. Lawley. On problems connected with item selection and test construction. Proceedings of the Royal Society of Edinburgh Section A: Mathematics, 61(3):273-287, 1943. URL https://doi . org/10. 1017/S0080454100006282. [p124]

X. Li, J. Zhang, and H.-h. Chang. Look-ahead content balancing method in variable-length computerized classification testing. British Journal of Mathematical and Statistical Psychology, 73(1):88-108, 2020. [p123]

S.-C. Ma, H.-H. Wang, and T.-W. Chien. A new technique to measure online bullying: online computerized adaptive testing. Annals of general psychiatry, 16(1):26, 2017. URL https: //doi.org/10.1186/s12991-017-0149-z. [p123]

D. Magis. A note on the equivalence between observed and expected information functions with polytomous irt models. Journal of Educational and Behavioral Statistics, 40(1):96-105, 2015. URL https://doi.org/10.3102/1076998614558122. [p124]

D. Magis and J. R. Barrada. Computerized adaptive testing with r: Recent updates of the package catr. Journal of Statistical Software, 76(1):1-19, 2017. URL https://doi .org/10.18637/ jss. v076. c01. [p123]

D. Magis, G. Raîche, et al. Random generation of response patterns under computerized adaptive testing with the r package catr. Journal of Statistical Software, 48(8):1-31, 2012. [p123]

P. Michel, K. Baumstarck, A. Loundou, B. Ghattas, P. Auquier, and L. Boyer. Computerized adaptive testing with decision regression trees: an alternative to item response theory for quality of life measurement in multiple sclerosis. Patient preference and adherence, 12:1043-1053, 2018. URL https://doi.org/10.2147/PPA. S162206. [p123]

A. Mizumoto, Y. Sasao, and S. A. Webb. Developing and evaluating a computerized adaptive testing version of the word part levels test. Language Testing, 36(1):101-123, 2019. URL https: //doi.org/10.1177/0265532217725776. [p123]

S. Nydick. catIrt: An R Package for Simulating IRT-Based Computerized Adaptive Tests, 2014. URL https://CRAN.R-project.org/package=catIrt. R package version 0.5-0. [p123]

M. W. Richardson. The relation between the difficulty and the differential validity of a test. Psychometrika, 1(2):33-49, 1936. URL https://doi .org/10.1007/BF02288003. [p124] 
J. Rodríguez-Cuadrado, D. Delgado-Gómez, J. C. Laria, and S. Rodríguez-Cuadrado. Merged tree-cat: A fast method for building precise computerized adaptive tests based on decision trees. Expert Systems with Applications, 143:113066, 2020. [p123, 124, 125]

F. Samejima. Estimation of latent ability using a response pattern of graded scores. Psychometrika monograph supplement, 34(4, Pt. 2), 1969. [p124, 127]

L. R. Tucker. Maximum validity of a test with equivalent items. Psychometrika, 11(1):1-13, 1946. URL https://doi.org/10.1007/BF02288894. [p124]

M. Ueno. Adaptive testing based on bayesian decision theory. In International Conference on Artificial Intelligence in Education, pages 712-716. Springer, 2013. [p123]

M. Ueno and P. Songmuang. Computerized adaptive testing based on decision tree. In 2010 10th IEEE International Conference on Advanced Learning Technologies, pages 191-193. IEEE, 2010. [p123]

W. J. Van der Linden and P. J. Pashley. Item selection and ability estimation in adaptive testing. In Elements of adaptive testing, pages 3-30. Springer, 2009. URL https://doi .org/10.1007/978-0-38785461-8_1. [p123]

W. J. Veerkamp and M. P. Berger. Some new item selection criteria for adaptive testing. Journal of Educational and Behavioral Statistics, 22(2):203-226, 1997. URL https://doi .org/10.3102/ 10769986022002203. [p123]

D. J. Weiss. Computerized adaptive testing for effective and efficient measurement in counseling and education. Measurement and Evaluation in Counseling and Development, 37(2):70-84, 2004. URL https://doi.org/10.1080/07481756.2004.11909751. [p123]

H.-M. Wu, B.-C. Kuo, and S.-C. Wang. Computerized dynamic adaptive tests with immediately individualized feedback for primary school mathematics learning. Educational Technology E Society, 20(1):61-72, 2017. URL https://www. jstor.org/stable/jeductechsoci.20.1.61. [p123]

D. Yan, C. Lewis, and M. Stocking. Adaptive testing with regression trees in the presence of multidimensionality. Journal of Educational and Behavioral Statistics, 29(3):293-316, 2004. [p123]

X. Zhou and M. D. Reckase. Optimal item pool design for computerized adaptive tests with polytomous items using gpcm. Psychological Test and Assessment Modeling, 56(3):255, 2014. [p123]

Z. H. ZhuoKang and Y. Liu. Computational intelligence and intelligent systems. 2012. [p123]

Javier Rodríguez-Cuadrado

Universidad Carlos III de Madrid, Department of Statistics

Leganés

Spain

(https://orcid.org/0000-0003-1315-8977)

javierro@est-econ.uc3m.es

Juan C. Laria

Universidad Carlos III de Madrid, Department of Statistics

Leganés

Spain

(https://orcid.org/0000-0001-7734-9647)

jlaria@est-econ.uc3m.es

David Delgado-Gómez

Universidad Carlos III de Madrid, Department of Statistics

Leganés

Spain

(https://orcid.org/0000-0002-2976-2602)

ddelgado@est-econ. uc3m.es 